\title{
Clinical and cost-effectiveness of a physiotherapy led case management service for back pain
}

- J. Smith,

- G. Yeowell,

- F. Fatoye

http://dx.doi.org/10.1016/j.physio.2016.10.151

Relevance: This evaluation of an innovative approach to the management of back pain offers opportunities for advancing physiotherapy and should promote physiotherapy to influence policy.

Purpose: Back pain is the most common musculoskeletal condition affecting $30 \%$ of the adult population in the United Kingdom (UK). It is associated with functional limitation, reduced quality of life (QoL), anxiety and depression, and is the leading cause of productivity loss. The management of back pain is resource intensive to the individuals, health service and society. Thus, new and innovative interventions that are clinically and cost-effective are constantly sought. In the private sector, a physiotherapy led Case Management (CM) service has been developed. The aim of this service is for clients to receive fast access to advice and the appropriate treatment, to reduce unnecessary diagnostics and surgical referrals, and reduce over-treatment cost. CM is relatively new to UK physiotherapists, as such, there is a lack of evidence regarding its clinical and cost-effectiveness.

Aim: To evaluate the clinical and cost-effectiveness of a UK private sector, physiotherapy CM service for back pain.

\section{Methods/analysis:}

Design: An observational cohort study was undertaken based on prospective data collection of patient-reported outcome measures; patient reported experience measure (PREM); and from an existing database.

Participants: Consecutive referrals into the CM service over a three-month period.

Interventions: Participants were managed within the service in accordance with usual pathways.

Outcome measures: QoL was examined using EQ-5D-5L. Functional limitation was assessed with Patient Specific Functional Scale [PSFS]. The PREM used was the Consultant and Relational Empathy [CARE] questionnaire. Measurements were taken at baseline and repeated at the point at which the client's case was closed. The CARE satisfaction questionnaire was administered on closure of the client's case.

A cost consequence analysis was completed using existing data for the same period of time in two separate years; 2011 (pre-CM) and 2014 (post-CM).

Data analysis: Descriptive analysis was undertaken. Changes between baseline and followup were assessed using Wilcoxon Signed Rank test. 
Results: 110 clients were referred to the study. Nine were excluded as they did not meet the inclusion criteria and three declined to participate. Baseline measures were collected from 98 participants, 36 were lost to follow-up and 62 completed the full set of outcome measures.

There was a significant improvement in QoL (EQ-5D-5L $P<0.0001$; EQ-VAS $P<0.001$ ) and functional ability $(P<0.001)$ following physiotherapy $\mathrm{CM}$. The CARE questionnaire showed high levels of patient satisfaction with $96 \%$ of members rating their case management as good to excellent. There was a cost reduction in therapy resource use from year 2011 to 2014 of $£ 252,842$ ( $£ 10,772,875$ to $£ 10,520,034$ respectively).

Discussion and conclusions: The physiotherapy CM service for back pain provided a significant improvement in QoL and functional outcomes, within efficient use of resources. Furthermore, service users were highly satisfied with their experience of this service.

Impact and implications: There is limited evidence of the clinical and cost effectiveness of a CM service for back pain. This is evaluation may help to demonstrate how physiotherapy can effectively impact and enhance this population's health and wellbeing.

Funding acknowledgement: No funding received. 